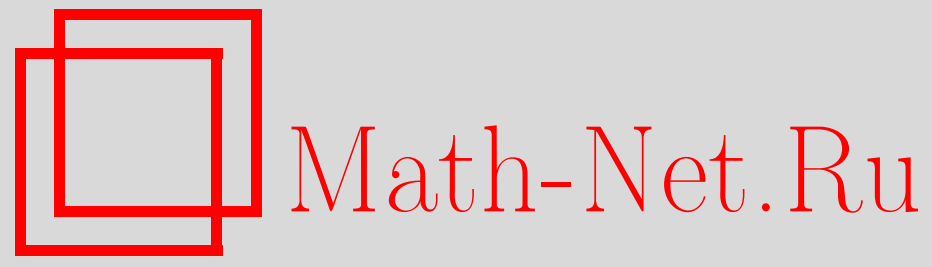

Ф. Айкарди, Самозацепление пространственных кривых без перегибов и его приложения, Функи. анализ и его прил., 2000, том 34, выпуск 2, 1-8

DOI: https://doi.org/10.4213/faa290

Использование Общероссийского математического портала MathNet.Ru подразумевает, что вы прочитали и согласны с пользовательским соглашением

http://www.mathnet.ru/rus/agreement

Параметры загрузки:

IP : 54.198 .64 .247

26 апреля 2023 г., 11:52:18

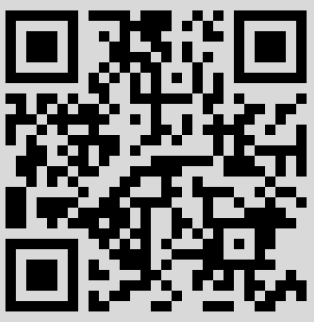


Функциональньй анализ и его приложения

2000, т. 34, вып. 2, с. 1-8

УДК 514.752 .23

\title{
Самозацепление пространственных кривых без перегибов и его приложения
}

\author{
(c) 2000. Ф. АЙКАРди
}

\section{Введение}

В 1959 г. Дж. Калугареану [1] ввел топологический инвариант гладких замкнутых кривых в евклидовом трехмерном пространстве. Этот инвариант называется самозацеплением кривой $\gamma$ и определяется как коэффициент зацепления кривой $\gamma$ с близкой кривой $\gamma_{\varepsilon}$, полученной из исходной кривой сдвигом каждой ее точки на $\varepsilon$ в направлении главной нормали к $\gamma$ в этой точке.

Калугареану получил формулу (типа гауссова интеграла) для самозацепления кривой в $\mathbb{R}^{3}$ без точек возврата и без кратных точек, заданной периодическими функциями $(x(s), y(s), z(s))$, имеющими непрерывные производные до порядка три. Он утверждал, что такое число - инвариант изотопии. Но это неверно по двум причинам:

1) В простейших точках перегиба репер Френе претерпевает скачкообразный поворот на угол $\pi$ вокруг направления касательной. Поэтому, если кривая $\gamma$ содержит точки перегиба, то кривая $\gamma_{\varepsilon}$ не является непрерывной и самозацепление не определено.

2) Значения самозацепления двух общих кривых без перегибов, которые находятся около кривой с перегибом, но с противоположных сторон от гиперповерхности кривых с одной точкой перегиба, не равны, хотя кривые изотопны.

В действительности самозацепление - это инвариант изотопии гладких замкнутых кривых, вложенных в $\mathbb{R}^{3}$, без точек перегиба. Ниже доказывается, что самозацепление является инвариантом Васильева порядка один: его скачок при пересечении гиперповерхности кривых с одним перегибом равен 1, а при пересечении гиперповерхности кривых с одной двойной точкой равен 2 .

Мы доказываем формулу, выражающую самозацепление кривой общего положения без точек перегиба через знаки двойных точек общей проекции на плоскость и знаки кручения в точках кривой, которые проектируются в точки перегиба.

Кроме того, мы показываем, что существуют локально выпуклые кривые (с ненулевыми значениями кривизны и кручения) с любым знаком кручения и любым значением самозацепления.

Самозацепление кривой, имеющей выпуклую проекцию на плоскость, равно нулю. Можно доказать, что отсюда вытекает известная теорема о четырех точках уплощения пространственной кривой, имеющей выпуклую проекцию на плоскость (см. $[2,3]$, а также обобщения этой теоремы в $[4,5])$.

Автор благодарен В. Д. Седых за многочисленные полезные замечания, позволившие существенно улучшить первоначальный вариант статьи. 


\section{$\S 1$. Самозацепление и его вычисление}

Кривизна гладкой иммерсии окружности в евклидово трехмерное пространство по определению неотрицательна. Точка, где кривизна обращается в нуль, называется точкой перегиба или просто перегибом.

Точка перегиба бывает только у иммерсии не общего положения, т. е. она исчезает при малом возмущении иммерсии в пространстве всех иммерсий. В простейшей точке перегиба, где кривизна имеет нуль первого порядка, кручение имеет ненулевое конечное значение.

Рассмотрим общее однопараметрическое семейство деформаций $c_{\alpha}$ кривой $c_{0}$, имеющей точку перегиба. В точке $p_{\alpha}$, отвечающей точке перегиба кривой $c_{0}$, кручение имеет минимум при $\alpha<0$ и его значение стремится к $-\infty$ при $\alpha \rightarrow 0$, а при $\alpha>0$ имеет максимум и его значение стремится к $\infty$ при $\alpha \rightarrow 0$. Это значит, что при $\alpha<0$ или при $\alpha>0$ кривая $c_{\alpha}$ имеет две точки уплощения в окрестности точки $p_{\alpha}$.

ЗАмеЧАниЕ. Разница между значениями интеграла кручения вдоль кривых $c_{-\alpha}$ и $c_{\alpha}$ стремится к $2 \pi$ при $\alpha \rightarrow 0$. (Калугареану [1], видимо, этого не заметил, хотя его формула самозацепления правильна.)

Пусть $\gamma$ - образ вложения окружности в трехмерное евклидово пространство без точек перегиба. Через $\gamma_{\varepsilon}^{b}$ (соответственно $\gamma_{\varepsilon}^{n}$ ) обозначим кривую, полученную из $\gamma$ сдвигом каждой точки на расстояние $\varepsilon$ в направление бинормали (нормали) к $\gamma$ в этой точке.

ОПРЕДЕЛЕНИЕ 1.1. Самозацеплением $K(\gamma)$ кривой $\gamma$ называется коэффициент зацепления $\left\{\gamma, \gamma_{\varepsilon}^{b}\right\}$.

ЗАмЕчАниЕ. $K(\gamma)=\left\{\gamma, \gamma_{\varepsilon}^{n}\right\}$ (гомотопия осуществляется скручиванием бинормали к нормали в нормальной плоскости). Самозацепление не зависит от выбора евклидовой метрики в $\mathbb{R}^{3}$. Оно меняет знак при изменении ориентации пространства $\mathbb{R}^{3}$.

Бинормаль меняется непрерывно при перемещениях кривой в множестве иммерсий без перегибов. Поэтому самозацепление - это инвариант вложенных кривых без перегибов. Менее очевидно, как самозацепление меняется при прохождении через кривую с перегибом.

ПрЕДЛОжЕНИЕ. СКачок самозачепления при прохождении через кривую с простейшим перегибом равен 1 (-1), если после прохождения две точки уплощения исчезают и в перегибе кручение положительно (отрицательно).

Предложение вытекает из формулы Калугареану (см. [1]); проиллюстрируем его следующим примером.

ОПРЕДЕЛЕНИЕ 1.2. Иммерсия окружности в трехмерное евклидово пространство называется локально выпуклой, если ее кручение и кривизна нигде не обращаются в нуль.

Рассмотрим замкнутые винтовые кривые $c_{r}^{m}$ на торе в $\mathbb{R}^{3}$, заданные в координатах уравнениями

$$
x=(1+r \sin (m t)) \cos (t), \quad y=(1+r \sin (m t)) \sin (t), \quad z=r \cos (m t),
$$

где $r<1$. Кривая $c_{r}^{m}$ локально выпукла, если $m>1$ и $r_{1}(m)<r<r_{2}(m)$ (см. $[6])$.

Например, при $m=3$ и $r=0.3$ кручение везде положительно, а при $r<$ $r_{1}(3)=0.1$ кривая $c_{r}^{3}$ имеет шесть точек уплощения. Действительно, кривая 
$c_{0.1}^{3}$ имеет три простейшие точки перегиба (при $t=\pi / 2,7 \pi / 6$ и $11 \pi / 6$ ), гдє кручение положительно. Самозацепление кривой $c_{r}^{3}$ при $r<0.1$ равно нулю, а при $r>0.1$ равно 3 (см. рис. 1). Скачок самозацепления равен 1 при прохождении через каждый перегиб.
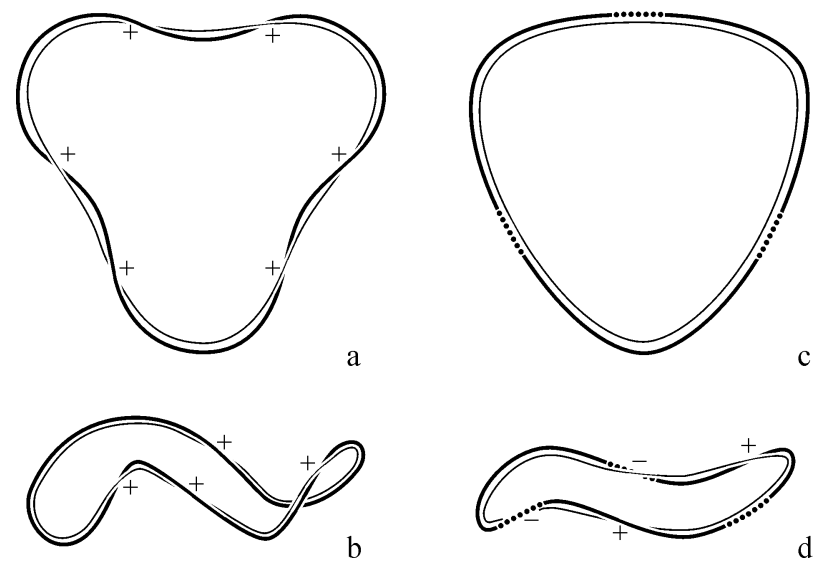

Рис. 1. а), b) проекции кривой $\left.\left.c_{0.3}^{3} ; \mathrm{c}\right), \mathrm{d}\right)$ проекции кривой $c_{0.09}^{3}$.

На изображенных точками дугах кручение отрицательно.

При отрицательных значениях $m$ получим кривые, являющиеся зеркальнс симметричными кривым с соответствующими положительными значениями $m$. Например, если $m=-3$, то самозацепление при $r<0.1$ равно нулю; при $r>0.1$ самозацепление равно -3 и кручение отрицательно.

В следующей теореме мы показываем, как можно вычислять значение самозацепления кривой общего положения без точек перегиба по ее проекции общегс положения на плоскость.

ОПРЕДЕЛЕНИЕ 1.3. Пусть $\left(c_{1}, P_{1}\right)$ и $\left(c_{2}, P_{2}\right)$ - ростки двух ориентированных кривых, вложенных в $\mathbb{R}^{3}$, проекции которых на плоскость П пересекаются в точке $p$. Пусть $v_{1}$ и $v_{2}$ - касательные векторы в $P_{1}$ и $P_{2}$ соответственно. Знак $s$ точки $p$ определяется таким образом: $s(p)=1$, если векторы $\left(v_{1}, v_{2}, P_{1}-P_{2}\right)$ положительно ориентируют $\mathbb{R}^{3} ;$ в противном случае $s(p)=-1$.

ЗАмЕчАниЕ. Знак точки не меняется при одновременном изменении ориентации обеих кривых.

ТЕОРемА. Пусть $p_{1}, \ldots, p_{n}$ - двойнье точки проекиии общего положения замкнутой гладкой кривой $\gamma$ общего положения без точек перегиба, $а f^{+} u f^{-}$ - числа точек перегиба проекции кривой $\gamma$, которые являются проекииями точек, где кручение положительно и отрицательно соответственно. Тогдо значение самозачепления задается формулой

$$
K(\gamma)=\frac{f^{+}-f^{-}}{2}+\sum_{i} s\left(p_{i}\right) .
$$

ДокАЗАТЕЛЬСтво. Легко проверить (см. [7]), что вклад в самозацеплениє каждой двойной точки проекции равен \pm 1 в зависимости от ее знака. Что касается первого члена, рассмотрим кривую $\gamma$, имеющую проекцию без двойных точек. Коэффициент зацепления кривых $\gamma$ и $\gamma_{\varepsilon}^{n}$ - это индекс пересечения кривой $\gamma_{\varepsilon}^{n}$ с любым полуцилиндром над кривой $\gamma$. Такой индекс пересечения 
равен половине вклада целого цилиндра, т. е. алгебраической суммы точек пересечения проекций кривых $\gamma$ и $\gamma_{\varepsilon}^{n}$. Точка пересечения проекций кривых $\gamma$ и $\gamma_{\varepsilon}^{n}$ стремится при $\varepsilon \rightarrow 0$ к точке перегиба проекции кривой $\gamma$. Действительно нормаль $n$ к $\gamma$ в точке $p$ принадлежит вертикальной плоскости, содержащей касательную к $\gamma$ в $p$ и направление проекции, только если такая плоскость соприкасающаяся плоскость кривой $\gamma$ в $p$. Знак точки пересечения проекций кривых $\gamma$ и $\gamma_{\varepsilon}^{n}$ равен знаку кручения в точке кривой $\gamma$, проекцией которой является точка перегиба. Теорема доказана.

ЗАмечАниЕ. Пара кривых $\gamma$ и $\gamma_{\varepsilon}^{n}$ составляет край ленты. Два члена суммы (2) соответствуют двум членам Twist и Write естественного инварианта изотопии ленточных узлов, называемого числом зацепления (см. $[8,9,10,11])$.

ПримеР. Применение формулы (2) к двум разным проекциям локально выпуклых кривых $c_{0.3}^{3}$ и $c_{0.09}^{3}$, показанным на рис. 1 , дает $K\left(c_{0.3}^{3}\right)=6 / 2$ (1a), $K\left(c_{0.3}^{3}\right)=4 / 2+1(1 \mathrm{~b}), K\left(c_{0.09}^{3}\right)=0(1 \mathrm{c}), K\left(c_{0.09}^{3}\right)=(2-2) / 2(1 \mathrm{~d})$.

Кручение выпуклой кривой $c_{r}^{m}$, заданной формулой $(1)\left(r_{1}(m)<r<r_{2}(m)\right.$ $|m|>1)$, положительно (отрицательно) при положительном (отрицательном) целом значении параметра $m$, и значение самозацепления равно $m$. Естественный вопрос: возможно ли, что локально выпуклая кривая с положительным кручением имеет отрицательное самозацепление? Ответ - да, возможно. Действительно, мы докажем следующее

ПРЕДЛОЖЕНИЕ. Существуют локально выпуклье кривые с любым знаком кручения и с любым значением самозачепления.

ДокАЗАТЕльство. Для каждого целого $m$ построим локально выпуклую кривую с положительным кручением, имеющую самозацепление, равное $m$.

Если $m>1$, мы имеем кривые $c_{r}^{m}\left(r_{1}(m)<r<r_{2}(m)\right)($ ср. $(1))$ с положительным кручением и с самозацеплением, равным $m$.

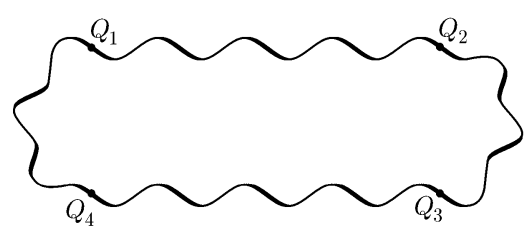

a

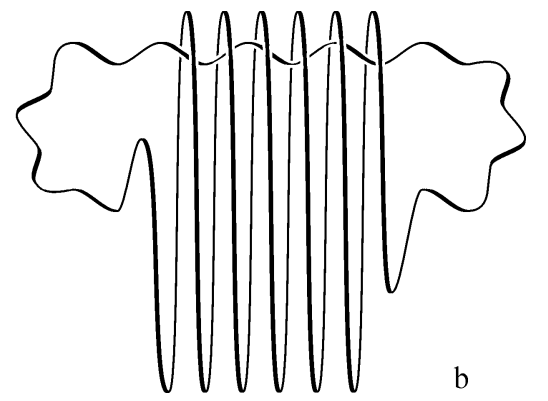

b

Рис. 2. а) проекция кривой с положительным кручением и самозацеплением, равным $14 ; \mathrm{b})$ проекция кривой с положительным кручением и самозацеплением, равным 6.

Если $m \leqslant 1$, вначале построим выпуклую кривую с положительным кручением и положительным самозацеплением. Такая кривая состоит из четырех дуг двух винтовых линий и двух половин локально выпуклой обмотки $c_{r}^{n}(n \geqslant 4$ четное) (см. рис. 2а). В окрестностях точек $Q_{1}, Q_{2}, Q_{3}$ и $Q_{4}$ дуги немного возмущены, чтобы кривая имела нужный порядок гладкости. Это можно сделать, сохраняя значение кручения положительным. Затем деформируем одну винтовую линию так, чтобы получить в ее центральной части винтовую линию с большим радиусом и меньшим шагом. Существуют гладкие деформации 
исходной винтовой линии, при которых значения кручения и кривизны постоянны в центральной части (оба меньше, чем у исходной дуги) и нигде не обращаются в нуль в остальных частях. Если радиус центральной винтовой линии достаточно большой, самозацепление деформированной кривой меньше, чем исходной. Действительно, в проекции кривой (см. рис. 2b) есть двойные точки, все с отрицательным знаком. Очевидно, что если шаг центральной винтовой линии уменьшается, то число двойных точек и число точек перегиба проекции увеличивается на одно и то же число. Но вклад в самозацепление одной пары двойных точек есть удвоенный вклад пары точек перегиба. Поэтому мы получаем кривые с любым отрицательным значением самозацепления.

\section{$\S 2$. Локальные инварианты пространственных кривых}

Здесь кривой называется отображение окружности в евклидово пространство $\mathbb{R}^{3}$, заданное в координатах уравнениями

$$
x=f_{1}(\theta), \quad y=f_{2}(\theta), \quad z=f_{3}(\theta), \quad \theta \in S^{1},
$$

где $f_{1}, f_{2}$ и $f_{3}$ - гладкие функции.

Кривые не общего положения образуют в пространстве всех кривых гиперповерхность, называемую дискриминантом. Инварианты Васильева - это локально постоянные функции, определенные на дополнении к дискриминанту.

Вне множества бесконечной коразмерности в пространстве всех кривых росток отображения (3) в окрестности каждой точки в надлежащей системе координат имеет вид (см. [12])

$$
x_{1}=t^{m}+\ldots, \quad x_{2}=t^{p}+\ldots, \quad x_{3}=t^{q}+\ldots,
$$

где $m<p<q$. Набор $(m, p, q)$ называется символом точки. Точка общего положения отображения (3) имеет символ $(1,2,3)$.

Кривая общего положения является вложенной и не имеет других точек не общего положения, кроме простейших точек уплощения (где кручение имеет нуль первого порядка, а кривизна положительна; их символ - $(1,2,4))$.

ОПрЕДЕЛЕНиЕ 2.1. Точка не общего положения, отличная от простейших точек уплощения, называется необычной.

Рассмотрим три части страта коразмерности один дискриминанта, соответствующие кривым, не имеющим других необычных точек, кроме одной точки одного из следующих типов:

cmpam $D$ содержит кривые с одной двойной точкой;

cmpam $B$ содержит кривые с одной точкой биуплощения (где кручение имеет нуль второго порядка; ее символ $(1,2,5))$;

cmpam $P$ содержит кривые с одной точкой перегиба (где кривизна имеет нуль первого порядка; ее символ $(1,3,4))$.

Объединение стратов $D, B$ и $P$ обозначим через $\Sigma$.

ЗАмЕчАниЕ. Кривая общего положения, а также кривая, принадлежащая $\Sigma$, иммерсированы.

ОПредЕЛЕниЕ 2.2. Инвариант гладких иммерсий окружности в $\mathbb{R}^{3}$ без необычных точек называется локальным, если его скачок при трансверсальном прохождении множества $\Sigma$ зависит лишь от поведения иммерсии в окрестности тех точек, которые при иммерсии попадают в необычную точку. 
Теорема. Каждый локальный инвариант кривых общего положения является, с точностью до аддитивной константьи, линейной комбиначией двух основных локальных инвариантов: числа точек уплощения и самозачепления.

ДоКАЗАТЕЛЬСтво. Подразделяем страты коразмерности один дискриминанта на следующие подстраты:

элемент страта $B$ принадлежит $B^{+}\left(B^{-}\right)$, если в окрестности точки биуплощения кручение неотрицательно (неположительно);

элемент страта $P$ принадлежит $P^{+}\left(P^{-}\right)$, если в окрестности точки перегиба кручение положительно (отрицательно);

элемент страта $D$ принадлежит $D^{++}\left(D^{--}\right)$, если в обеих окрестностях двойной точки кручение положительно (отрицательно), и $D^{+-}$, если в одной окрестности двойной точки кручение положительно, а в другой отрицательно.

ОПРЕДЕЛЕниЕ 2.3. Локальная коориентация страта коразмерности один в точке - это выбор одной из двух частей дополнения к дискриминанту в окрестности этой точки, локально отделенных этим стратом. Выбранная часть называется положительной, а другая - отричательной. Трансверсальное прохождение через локально коориентированный страт от отрицательной к положительной стороне называется положительным.

Коориентируем страты $B, P$ и $D$ следующим образом:

Страт $B$. Положительной считается сторона страта $B^{+}$(или $B^{-}$), где число точек уплощения больше.

Страт $P$. Положительной считается сторона страта $P^{+}$(или $P^{-}$), где число точек уплощения меньше.

Страт D. Рассмотрим две кривые общего положения, которые принадлежат однопараметрическому семейству, пересекающему страт $D$ в кривой $\gamma_{d}$ с двойной точкой $d$ и находятся около $\gamma_{d}$ с противоположных сторон от этого страта. Проекции этих кривых на плоскость, содержащую двойную точку $d$ и обе касательные к $\gamma_{d}$ в этой точке, имеют двойную точку около $d$. Знаки (см. определение 1.3) таких двойных точек противоположны. Положительной считается сторона страта $D^{++}\left(D^{--}\right.$или $\left.D^{+-}\right)$, где знак положителен.

ОБозначение. Через $\left.\Delta(I)\right|_{S}$ обозначается значение локального скачка функции $I$, принимающей целые значения на дополнении к дискриминанту, при положительном пересечении страта $S\left(S=B^{+}, B^{-}, P^{+}\right.$и т.д.).

Локальные скачки $\left.\Delta(I)\right|_{S}$ определяют, с точностью до аддитивной константы, локальный инвариант $I$ кривых общего положения, если и только если

$$
\Delta(I)=\left.\sum_{S} n(S) \Delta(I)\right|_{S}=0
$$

для любого замкнутого пути в пространстве всех кривых, где $n(S)$ равно алгебраическому числу пересечений локального страта $S$.

Замкнутые пути, зацепленные со стратами коразмерности два замыкания множества $\Sigma$, доставляют необходимые условия существования локальных инвариантов.

ОПРЕДЕЛЕниЕ 2.4. Страт коразмерности два называется тривиальным, если он является трансверсальным пересечением двух гладких ветвей замыкания множества $\Sigma$.

Тривиальные страты содержат кривые, которые не имеют других необычных точек, кроме двух различных двойных точек или двух различных точек 
биуплощения, или одной двойной точки и одной точки биуплощения (не совпадающей с двойной точкой) и т. д. В окрестности тривиального страта коориентации двух ветвей одного и того же страта коразмерности один согласованы. Следовательно, условие коцикла тривиальным образом выполняются.

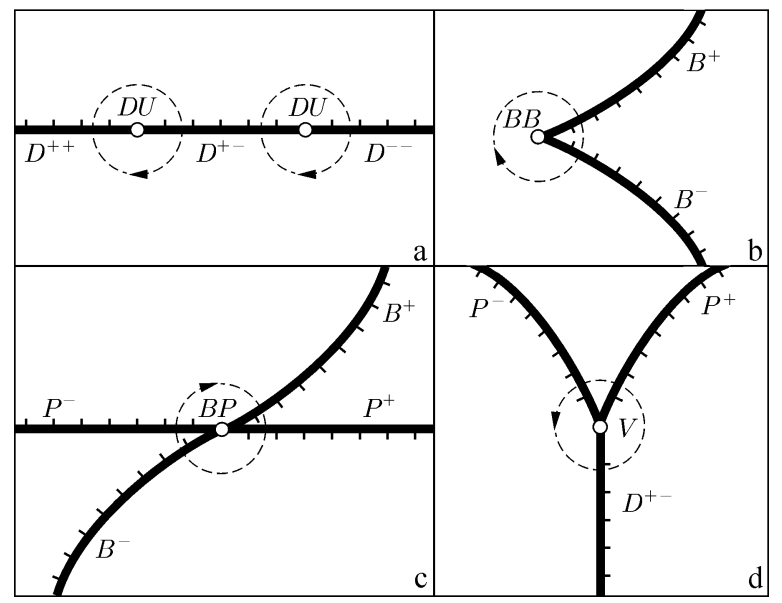

Рис. 3. Бифуркационные диаграммы стратов коразмерности два.

Нетривиальные страты коразмерности два, которые находятся в замыкании множества $\Sigma$, перечислены в следующем списке, где также указаны соответствующие уравнения:

Cmpam DU содержит кривые, которые не имеют других необычных точек. кроме одной двойной точки, совпадающей с общей точкой уплощения. Получаем уравнения (см. рис. $3 \mathrm{a})$

$$
\begin{aligned}
& \left.\Delta(I)\right|_{D^{++}}-\left.\Delta(I)\right|_{D^{+-}}=0, \\
& \left.\Delta(I)\right|_{D^{+-}}-\left.\Delta(I)\right|_{D^{--}}=0 .
\end{aligned}
$$

$C$ mam $B B$ содержит кривые, не имеющие других необычных точек, кроме одной не общей точки уплощения, где кручение имеет нуль третьего порядка (символ $(1,2,6))$. Получается уравнение (см. рис. $3 \mathrm{~b})$

$$
\left.\Delta(I)\right|_{B^{+}}-\left.\Delta(I)\right|_{B^{-}}=0 .
$$

Cmpam BP содержит кривые, не имеющие других необычных точек, кроме одной точки, где и кривизна, и кручение имеют нуль первого порядка (символ $(1,3,5))$. Получается уравнение (см. рис. 3c)

$$
\left.\Delta(I)\right|_{B^{+}}+\left.\Delta(I)\right|_{P^{+}}+\left.\Delta(I)\right|_{B^{-}}+\left.\Delta(I)\right|_{P^{-}}=0 .
$$

Cmpam $V$ содержит неиммерсированные кривые, не имеющие других необычных точек, кроме одной простейшей точки возврата (символ $(2,3,4))$. Получается уравнение (см. рис. $3 \mathrm{~d}$ )

$$
\left.\Delta(I)\right|_{D^{+-}}-\left.\Delta(I)\right|_{P^{+}}+\left.\Delta(I)\right|_{P^{-}}=0 .
$$

ЗАмЕчАниЕ. Бифуркационные диаграммы, показанные на рисунках $3 \mathrm{~b}, 3 \mathrm{c}$ и $3 \mathrm{~d}$, получены из 2-параметрических версальных деформаций в 0 отображений b) $x=t, y=t^{2}, z=t^{6}$; c) $x=t, y=t^{3}, z=t^{5}$; d) $x=t^{2}, y=t^{3}, z=t^{4}$. Такиє 
кривые в нуле просты, т. е. все необычные точки близких кривых принадлежат конечному набору классов эквивалентности (не имеют модулей).

Ранг линейной системы (4)-(8) равен 5. Два линейно независимых решения, т. е. два набора значений локальных скачков $\Delta(I)_{S}$, приводятся в таблице (для простоты обозначим через $D$ все три подстрата двойной точки):

\begin{tabular}{|c|c|c|c|c|c|}
\hline$I$ & $\left.\Delta(I)\right|_{B^{+}}$ & $\left.\Delta(I)\right|_{B^{-}}$ & $\left.\Delta(I)\right|_{P^{+}}$ & $\left.\Delta(I)\right|_{P^{-}}$ & $\left.\Delta(I)\right|_{D}$ \\
\hline$F$ & 2 & 2 & -2 & -2 & 0 \\
\hline$K$ & 0 & 0 & 1 & -1 & 2 \\
\hline
\end{tabular}

Очевидно, что $\Delta(K)$ - это скачок самозацепления, а $\Delta(F)$ - скачок числа точек уплощения. Существование интегральных формул для инвариантов $F$ и $K$ гарантирует апостериори, что коциклы, определенные скачками $\Delta(F)$ и $\Delta(K)$, тривиальны. Теорема доказана.

ЗАмЕчАниЕ. Локальные инварианты являются по определению инвариантами Васильева порядка один. Я не знаю нелокальных инвариантов порядка один замкнутых пространственных кривых.

\section{ЛитеРАТУРА}

1. Calugareanu G. L'integrale de Gauss et I'analyse des noeuds tridimensionnels. Rev. Math. Pures Appl., 4, No. 1, 5-20 (1959).

2. Barner $M$. Über die Mindestanzahl stationärer Schmiegebenen bei geschlossen strengkonvexen Raumkurven. Abh. Math. Sem. Univ. Hamburg, 20, 196-215 (1956).

3. Arnold $V$. I. On the number of flattening points on space curves. Institut MittagLeffler, Report no. 1 (1994-1995), pp. 1-13.

4. Седbı В. Д. Теорема о четырех вершинах выпуклой пространственной кривой. Функц. анализ и его прил., 26, вып. 1, 35-41 (1992).

5. Romero Fuster M. C., Sedykh V. D. A lower estimate for the number of zero-torsion points of a space curve. Beiträge Algebra Geom., 38, No. 1, 183-192 (1997).

6. Rodrigues Costa Sueli I. On closed twisted curves, Proc. Amer. Math. Soc., 109, No. 1, 205-214 (1990).

7. Aicardi F. Topological invariants of knots and framed knots in the solid torus. C. R. Acad. Sci. Paris Sér. I, 321, 199-204 (1995).

8. White J. H. Self-linking and Gauss integral in higher dimensions. Amer. J. Math., 91, 693-728 (1969).

9. Fuller F. B. Decomposition of the linking number of a closed ribbon: a problem from molecular biology. Proc. Nat. Acad. Sci. U.S.A., 75, No. 8, 3557-3561 (1978).

10. Kauffman L. H. Knots and Physics. Word Scientific, Singapore, 1991, pp. 488-489.

11. Arnold V. I., Khesin B. A. Topological Methods in Hydrodinamics. Applied Mathematical Sciences, Vol. 125, Springer-Verlag, 1998, pp. 177-179.

12. Арнольд В. И. Топологические вопросы теории асимптотических кривых. Труды МИАН, 225 (1999).

Scuola Internazionale Superiore di Studi Avanzati, Trieste, Italy

Поступило в редакцию 28 декабря 1998 г. 